\title{
Properties of holographic mesons on dense medium
}

\author{
Bum-Hoon Lee, ${ }^{a, b}$ Chanyong Park ${ }^{b}$ and Siyoung Nam ${ }^{a}$ \\ ${ }^{a}$ Department of Physics, Sogang University, \\ Seoul, 121-742 Korea \\ ${ }^{b}$ CQUeST, Sogang University, \\ Seoul, 121-742 Korea \\ E-mail: bhl@sogang.ac.kr, cyong21@sogang.ac.kr, stringphy@gmail.com
}

ABStract: We study the energy dispersions of holographic light mesons and their decay constants on dense nuclear medium. As the spatial momenta of mesons along the boundary direction increase, both observables of the mesons not only increase but also split according to the isospin charges. The decay constant of the negative meson is more large than that of the positive meson of the same type due to the chemical potentials of the background nucleons.

KEYwords: Gauge-gravity correspondence, AdS-CFT Correspondence, Holography and condensed matter physics (AdS/CMT)

ARXiv EPRINT: 1412.3097 


\section{Contents}

$\begin{array}{llr}1 & \text { Introduction } & 1\end{array}$

2 Backgrounds for confinement at zero temperature $\quad 2$

3 Dispersion relation and decay constant of the $\rho$ meson $\quad 6$

4 Dispersion relation and decay constant of the $a_{1}$ meson 9

5 Dispersion relation and decay constant of the $\pi$ meson $\quad 11$

$\begin{array}{llr}6 & \text { Discussion } & 14\end{array}$

\section{Introduction}

During the last decade, one of the important developments in theories of the high energy physics is the AdS/CFT correspondence [1-3], which provides a map between string theories on Anti-de Sitter(AdS) and conformal field theories(CFT) living on the boundary of the AdS. Motivated by the success of the AdS/CFT correspondence, many generalizations to maps between gravity theories and quantum field theories(QFT), especially quantum chromodynamics(QCD), has been proposed and tested under the notion of the gauge/gravity duality. Usually, QFT calculations are performed by perturbation theory and this makes it inaccessible to capture the physics in the strongly-coupled regime of QFT's. In that sense, the gauge/gravity duality is extremely useful because we use a weakly-coupled gravity theory to describe dynamics of a strongly-coupled QFT. For example, the confinement in a hadronic state is a non-perturbative phenomenon, which makes it difficult to study the physics of it. However, one of the recent applications of the gauge/gravity duality, the so-called AdS/QCD sheds light on studying mesons in the confinement phase.

In this work, we will be interested in the hard-wall AdS/QCD model $[4,5,9]$. This is a bottom-up approach to QCD whose goal is to reproduce known properties of QCD by choosing an appropriate five-dimensional asymptotic AdS gravity. In this model, it is possible to incorporate chiral symmetry breaking by a bi-fundamental bulk scalar field containing quark mass and chiral condensate [8]. Moreover, a sharp cut-off, called hard-wall, located at the finite bulk radial coordinate $z=z_{\mathrm{IR}}$ generates the mass gap and confinement $[6,7]$. Some static hadronic observables such as masses, decay constants, charge radius, and form factors have been studied and reproduced in the hard-wall AdS/QCD model $[4,5,10-16]$.

These works can be further generalized to the bottom-up models on dense medium [1728]. The density in the dual boundary field theory implies to introduce a $U(1)$ gauge 
field in the bulk AdS gravity action. Then, the well-known solution is given by ReissnerNordstrom AdS (RN AdS) black hole, from which thermal charged AdS(tcAdS) space can be constructed to study confining phase of the dense medium at zero temperature, which is nothing but RN AdS black hole of vanishing mass with a hard-wall [19]. The inserted hard-wall plays a role of setting up an infrared cutoff of the dual boundary theory in the confining phase and, at the same time, prevents any harm to bulk quantities from the naked singularity. The generalization to the two-flavour $U(2)$ case is also possible where combinations of two Cartans are mapped to the chemical potentials or number densities of $u$ and $d$ quark [25]. Since the geometry corresponds to confining phase of the boundary theory, the fundamental excitations are not quarks but nucleons. Appropriate combinations of such bulk gauge fields were reinterpreted as the density of nucleons and this geometry was used to study meson masses and binding energy of a heavy quarkonium in nuclear matter [17].

In this paper, we will work in the same background used in [17] in order to study the dependence of dispersion and decay constant of mesons on the background nucleon density and their spatial momenta along the boundary. Like in the mass spectrum, if the spatial momenta increase, the non-trivial splitting and increasing of meson energies are developed due to the flavour charges of the background nucleons. As for decay constant of the mesons, the similar behaviors are observed but the decay constant of negative meson is more large than that of positive meson of the same type.

The paper is organized in the following way. We briefly introduce the tcAdS with a hard-wall and present the Lagrangian of the fluctuations in section 2. The numerical analysis of vector mesons is given in section 3 . The cases of the axial-vector and pions are discussed in section 4 and 5, respectively. In the final section VI, we summarize the paper.

\section{Backgrounds for confinement at zero temperature}

In the hardwall model of AdS/QCD, either a pure AdS space or its Euclidean version, thermal AdS(tAdS) space, is an useful geometry for studying the physics of confinement phase. Since we want to consider mesons on a dense medium in confining phase, some gauge fields dual to a dense medium must be included in bulk gravity action. Then, the thermal charged $\mathrm{AdS}(\mathrm{tcAdS})$ space can be one of the useful candidates. To construct a background geometry, it is necessary to identify two quantum numbers, baryon and isospin charges [25]. For this purpose, we choose the bulk gauge group to be $U(2)_{L} \times U(2)_{R}$, which realizes the $N_{f}=2$ lightest flavor symmetry of the dual theory. An action suitable for this is given by (See the details in $[17,25]$ )

$$
\begin{aligned}
S=\int d^{5} x \sqrt{-G}\left[\frac{1}{2 \kappa^{2}}(\mathcal{R}-2 \Lambda)-\frac{1}{4 g^{2}} \operatorname{Tr}\left(F_{M N}^{(L)} F^{(L) M N}\right.\right. & \left.+F_{M N}^{(R)} F^{(R) M N}\right) \\
& \left.+\left|D_{M} \Phi\right|^{2}+m^{2}|\Phi|^{2}\right] .
\end{aligned}
$$

The gauge group can be further decomposed into $S U(2)_{V} \times S U(2)_{A} \times U(1)_{V} \times U(1)_{A}$ in terms of the vector and axial subgroups. In this paper, we concentrate on the vector subgroups because they clarify the quantum numbers of the lowest nucleons. According to the 
gauge/gravity duality, the boundary value of the $U(1)$ vector corresponds to the chemical potential of the quark. In addition, we keep only the Cartan of $S U(2)_{V}$, whose boundary value is mapped to the isospin chemical potential. If turning on the time components of the $U(1) \times U(1)$, the action for our background solution is

$$
\begin{aligned}
S=\int d^{5} x \sqrt{-G}[ & \frac{1}{2 \kappa^{2}}(\mathcal{R}-2 \Lambda) \\
& \left.\quad-\frac{1}{4 g^{2}}\left(F_{M N}^{(u)} F^{(u) M N}+F_{M N}^{(d)} F^{(d) M N}\right)+\left|D_{M} \Phi\right|^{2}+m^{2}|\Phi|^{2}\right],
\end{aligned}
$$

where the cosmological constant is $\Lambda=-6 / R^{2}$ and $m^{2}=-3 / R^{2}$. From now on, we set the radius of the curvature $R$ unity. The gauge coupling $g$ and the gravitational coupling $\kappa$ are related to the rank $N_{c}$ of the color group via,

$$
g=\sqrt{\frac{4 \pi^{2}}{N_{c}}} \quad \text { and } \quad \kappa=\sqrt{\frac{4 \pi^{2}}{N_{c}^{2}}} .
$$

The field strength of the background gauge field $A_{M}^{\alpha}(\alpha=u, d)$ is defined by

$$
F_{M N}^{(\alpha)}=\partial_{M} A_{N}^{\alpha}-\partial_{N} A_{M}^{\alpha}
$$

and the covariant derivative acting on the complex scalar field $\Phi$ is defined by

$$
D_{M} \Phi=\partial_{M} \Phi-\frac{i}{\sqrt{2}}\left(A_{M}^{(u)}+A_{M}^{(d)}\right) \Phi+\frac{i}{\sqrt{2}} \Phi\left(A_{M}^{(u)}+A_{M}^{(d)}\right) .
$$

The equations of motion derived from the action (2.2) consist of Einstein-Maxwell and scalar equations. We will solve this system without considering any gravitational backreaction. A general solution to the Einstein-Maxwell equations is given as a wellknown Reissner-Nordström AdS (RN AdS) black hole. There exist two zero-temperature limits of the RN AdS black hole such as an extremal RN AdS black hole and a tcAdS geometry. To work in confinement phase of dual boundary theory, we introduce a hardwall in the bulk, which makes the radial coordinate $z$ to run from the boundary $z=0$ to the hard wall $z=z_{I R}$. It turns out that, within a hardwall formalism, tcAdS is more preferable to confinement in low density and low temperature regime than the usual extremal RN AdS black hole [19]. The resulting tcAdS solution merely takes the form of the zero-temperature limit of the RN AdS black hole of vanishing mass with the inserted hardwall, which hides a naked singularity. This hardwall also breaks the scaling symmetry in the bulk, which, in turn, implies the broken conformal symmetry, i.e. setting up an IR cutoff in the dual boundary theory according to the gauge/gravity duality. It is a well-known fact that fourdimensional QCD in low energy scale is in the phase of confinement. Now, we present the tcAdS solution,

$$
d s^{2}=\frac{1}{z^{2}}\left(-f(z) d t^{2}+\frac{1}{f(z)} d z^{2}+d \vec{x}^{2}\right)
$$

with

$$
f(z)=1+\sum_{\alpha=u, d} q_{\alpha}^{2} z^{6}
$$


while leaving the details in the explanations of the works [17, 25]. The gauge field is given by

$$
A_{0}^{\alpha}=2 \pi^{2} \mu_{\alpha}-Q_{\alpha} z^{2},
$$

where the chemical potential and the number density of the quarks are denoted as $\mu_{\alpha}$ and $Q_{\alpha}$, which are related to each other in tcAdS space by

$$
q_{\alpha}^{2}=\frac{2 \kappa^{2}}{3 g^{2}} Q_{\alpha}^{2}
$$

Moreover, after an appropriate holographic renormalization, to guarantee the well-defined Legendre transformation of the thermodynamic energies $[19,20,23,25]$ leads to the relation,

$$
Q_{\alpha}=\frac{3 \pi^{2}}{z_{\mathrm{IR}}^{2}} \mu_{\alpha}
$$

Since the number densities of $\mathrm{u}$ and $\mathrm{d}$ quarks corresponding to bulk gauge fields are conserved and we are in the confinement phase, then judicious recombination of bulk gauge fields may be reinterpreted as those of nucleons. This can be justified by observing that turning off any dense effect reproduces the known mass spectrum and value of decay constant of mesons in [4]. So it is convenient to write the background solution in terms of the nucleons, not of the quarks, because we are mainly interested in the confining phase on a dense medium. If we denote $Q_{P}$ and $Q_{N}$ as number densities of the proton and the neutron, then these are correlated with the corresponding quantities of the quarks by the relation $Q_{u}=2 Q_{P}+Q_{N}$ and $Q_{d}=Q_{P}+2 Q_{N}$. For practical purpose, it is convenient to use the total number density $Q$ and the density difference $D$ between the nucleons defined by

$$
Q=Q_{P}+Q_{N} \quad \text { and } \quad D=Q_{P}-Q_{N}
$$

It is also useful to define a relation $D=\alpha Q$ where the range for $\alpha$ is given by $-1 \leq \alpha \leq 1$. If $\alpha=0$, then the background density originates from equal numbers of the protons and the neutrons. A nuclear matter which is composed of only one species, either the proton or the neutron, implies $\alpha= \pm 1$. With this, we can rewrite the metric of the tcAdS space by

$$
d s^{2}=\frac{R^{2}}{z^{2}}\left(-f d t^{2}+\frac{d z^{2}}{f}+d \vec{x}^{2}\right)
$$

where

$$
f=1+\frac{3 Q^{2} \kappa^{2}}{g^{2}} z^{6}+\frac{D^{2} \kappa^{2}}{3 g^{2}} z^{6}
$$

and the gauge field by

$$
\begin{aligned}
& \bar{V}_{t}^{0}=\frac{A_{t}^{u}+A^{d}}{\sqrt{2}}=\frac{Q}{\sqrt{2}}\left(2 z_{I R}^{2}-3 z^{2}\right), \\
& \bar{V}_{t}^{3}=\frac{A_{t}^{u}-A^{d}}{\sqrt{2}}=\frac{D}{3 \sqrt{2}}\left(2 z_{I R}^{2}-3 z^{2}\right) .
\end{aligned}
$$


On the other hand, the background scalar field $\bar{\phi}$, the modulus of the scalar field $\Phi$, is obtained by solving the equation

$$
\frac{1}{\sqrt{-G}} \partial_{z}\left(\sqrt{-G} \partial^{z} \bar{\phi}\right)+3 \bar{\phi}=0
$$

on the background of tcAdS space described by the metric (2.12), without considering any gravitational back-reaction $[4,5,17]$. In general, the gravitational backreaction of the scalar field affects the background geometry and corresponds to $1 / N_{c}$ correction, which is very small [35]. The field $\bar{\phi}$ describes the chiral symmetry breaking and the analytic solution is given by

$$
\bar{\phi}=m_{q} z_{2} F_{1}\left(\frac{1}{6}, \frac{1}{2}, \frac{2}{3},-\frac{\left(D^{2}+9 Q^{2}\right)}{3 N_{C}} z^{6}\right)+\sigma z^{3}{ }_{2} F_{1}\left(\frac{1}{2}, \frac{5}{6}, \frac{4}{3},-\frac{\left(D^{2}+9 Q^{2}\right)}{3 N_{C}} z^{6}\right),
$$

where $m_{q}$ represents a quark mass and $\sigma$ a chiral condensate.

Before moving on the topic about the fluctuations on this background, we pose to mention two interesting possible backgrounds. The first case corresponds to isospin matter with two flavours [17, 29-33]. When referring to [17], this is described by setting $Q_{\alpha}=0$ in original bulk gauge field written in terms of quarks $(\alpha=u, d)$, and results in constant gauge fields, $\bar{V}_{t}^{3}=\sqrt{2} \pi^{2}\left(\mu_{\mathrm{P}}-\mu_{\mathrm{N}}\right)$ and $\bar{V}_{t}^{0}=0$, in the confining phase where $\mu_{\mathrm{P}}$ and $\mu_{\mathrm{N}}$ are isospin chemical potentials of proton $\mathrm{P}$ and neutron $\mathrm{N}$, respectively. The mass formula for mesons on the isospin matter shows the clear patterns of splitting dependent on the isospin charges. The second example obtained by setting $Q \neq 0$ and $\alpha=0$ (or $D=0$ ) describes a degenerate case, because the parameter $D$ explains the effect of isospins and $Q$ the one of background densities. This can be easily seen by examining equations of motion with respect to mesons, which will be presented in the latter part of the paper. All the equations for each meson take the same form, solely the one of neutral mesons.

Now, we turn on various fluctuations on the tcAdS background to investigate dispersion relations and decay constants of light mesons in the confining phase. The fluctuations $l_{M}^{a}$, $r_{M}^{a}$ and $\pi^{a}$ are defined through

$$
\begin{aligned}
L_{M}^{a} & =\bar{V}_{M}^{a}+l_{M}^{a}, \\
R_{M}^{a} & =\bar{V}_{M}^{a}+r_{M}^{a}, \\
\Phi & =\bar{\phi} e^{i \sqrt{2} \pi},
\end{aligned}
$$

where the barred fields denote the background fields. Nonzero values of $\bar{V}_{t}^{0}$ and $\bar{V}_{t}^{3}$ satisfying the equations of motion derived from the action (2.2) represent the nucleon's number density and the net isospin charge of a nuclear medium respectively [17, 25], whereas fluctuations, $l_{M}^{a}, r_{M}^{a}$ and $\pi^{a}$ describe various mesons of the dual field theory.

Since the $U(1)$ subsector corresponding to $l_{M}^{0}, r_{M}^{0}$ and $\pi^{0}$ decouples from the other sector, we can solve it independently. However, in this paper we focus on the $S U(2)$ sector with gauge index $i=1, \ldots, 3$. Vector meson $v_{\mu}^{i}$ and axial-vector meson $a_{\mu}^{i}$ of the dual field theory are introduced by redefining vector fluctuations as

$$
l_{\mu}^{i}=\frac{v_{\mu}^{i}+a_{\mu}^{i}}{\sqrt{2}} \text { and } \quad r_{\mu}^{i}=\frac{v_{\mu}^{i}-a_{\mu}^{i}}{\sqrt{2}} .
$$


At this stage of the Lagrangian to the quadratic order, there exist mixing terms between the axial-vector $a_{\mu}^{i}$ and the pseudoscalar $\pi^{i}$, which can be removed by decomposing the axial-vector field into the transverse piece $\bar{a}_{\mu}^{i}$ and the longitudinal piece such as [4]

$$
a_{\mu}^{i}=\bar{a}_{\mu}^{i}+\partial_{\mu} \chi^{i}
$$

subject to the divergenceless condition $\partial_{\mu} \bar{a}^{i \mu}=0$. As in [17], we set $v_{t}^{i}=\bar{a}_{t}^{i}=0$ under the Coulomb-like gauge fixings. More precisely, we take $v_{z}=0$ and $\partial^{\mu} v_{\mu}=0$ for vector mesons, whereas $a_{z}=0$ and $\partial^{\mu} a_{\mu}^{i}=\partial^{\mu} \partial_{\mu} \chi^{i}$ with $\chi^{i}$ satisfying $a_{t}^{i}=\partial_{t} \chi^{i}$ are taken for axial vectors. Under this gauge fixing, $v_{t}^{i}=0$ becomes a trivial solution satisfying the equation of motion. Due to the mixing between axial vectors and pions, on the other hand, we imposed a slightly different gauge fixing for axial vectors in which since $a_{t}^{i}$ is absorbed into the gauge transformation function, $\chi$, we can set $\bar{a}_{t}^{i}=0$. As a consequence, only $l_{m}^{i}, r_{m}^{i}$, and $\pi^{i}$ survive in the resulting fluctuation action

$$
\mathcal{L}_{2}^{(2)}=\mathcal{L}_{v}+\mathcal{L}_{a}+\mathcal{L}_{\pi \chi},
$$

with

$$
\begin{aligned}
4 g^{2} \mathcal{L}_{v}= & \partial_{M} v_{n}^{i} \partial^{M} v^{i n}+\bar{V}_{t}^{3} \bar{V}^{3 t}\left(v_{m}^{i} v^{i m}-v_{m}^{3} v^{3 m}\right)-2 \epsilon_{3 i j} \bar{V}^{3 t} \partial_{t} v_{m}^{i} v^{j m}, \\
4 g^{2} \mathcal{L}_{a}= & \partial_{M} \bar{a}_{n}^{i} \partial^{M} \bar{a}^{i n}+\bar{V}_{t}^{3} \bar{V}^{3 t}\left(\bar{a}_{m}^{i} \bar{a}^{i m}-\bar{a}_{m}^{3} \bar{a}^{3 m}\right)+4 g^{2} \phi^{2} \bar{a}_{m}^{i} \bar{a}^{i m}-2 \epsilon_{3 i j} \bar{V}^{3 t} \partial_{t} \bar{a}_{m}^{i} \bar{a}^{j m}, \\
4 g^{2} \mathcal{L}_{\pi \chi}= & \partial_{z} \partial_{\mu} \chi^{i} \partial^{z} \partial^{i} \chi^{i}+\bar{V}_{t}^{3} \bar{V}^{3 t}\left(\partial_{m} \chi^{i} \partial^{m} \chi^{i}-\partial_{m} \chi^{3} \partial^{m} \chi^{3}\right)+4 g^{2} \phi^{2}\left[\partial_{\mu} \chi^{i} \partial^{\mu} \chi^{i}\right. \\
& \left.+\partial_{M} \pi^{i} \partial^{M} \pi^{i}+\bar{V}_{t}^{3} \bar{V}^{3 t}\left(\pi^{i} \pi^{i}-\pi^{3} \pi^{3}\right)-2 \partial_{\mu} \chi^{i} \partial^{\mu} \pi^{i}-2 \epsilon_{3 i j} \bar{V}^{3 t} \pi^{i} \partial_{t}(\chi-\pi)^{j}\right] .
\end{aligned}
$$

\section{Dispersion relation and decay constant of the $\rho$ meson}

We study low energy modes of the vector fluctuations, which are identified as the states of $\rho$ mesons. For the purpose of it, we define

$$
\rho_{m}^{0}=v_{m}^{3} \quad \text { and } \quad \rho_{m}^{ \pm}=\frac{1}{\sqrt{2}}\left(v_{m}^{1} \pm i v_{m}^{2}\right)
$$

where the superscript 0 and \pm represent their isospin charges. In terms of these, the Lagrangian of the vector fluctuation is rewritten as

$$
2 g^{2} \cdot \mathcal{L}_{v}=\frac{1}{2} \partial_{M} \rho_{n}^{0} \partial^{M} \rho^{0 n}+\partial_{M} \rho_{n}^{+} \partial^{M} \rho^{-n}+\bar{V}_{t}^{3} \bar{V}^{3 t} \rho_{m}^{+} \rho^{-m}+i \bar{V}_{t}^{3}\left(\rho_{m}^{+} \partial^{t} \rho^{-m}-\rho_{m}^{-} \partial^{t} \rho^{+m}\right) .
$$

It is convenient to work in the momentum representation and we write the fourdimensional Fourier-transforms of $\rho$-mesons as

$$
\begin{aligned}
& \rho_{n}^{0}(x, z)=\int \frac{d^{4} p}{(2 \pi)^{4}} \tilde{\rho}_{n}^{0}(p, z) e^{i\left(\vec{p} \cdot \vec{x}-w_{0} t\right)}, \\
& \rho_{n}^{ \pm}(x, z)=\int \frac{d^{4} p}{(2 \pi)^{4}} \tilde{\rho}_{n}^{ \pm}(p, z) e^{i\left(\vec{p} \cdot \vec{x}-w_{ \pm} t\right)},
\end{aligned}
$$

where $p \equiv \sqrt{\vec{p}^{2}}$ is the magnitude of the spatial momenta in the boundary space. The $w_{0}$ and $w_{ \pm}$can be identified with mass terms of the neutral meson and charged ones only 


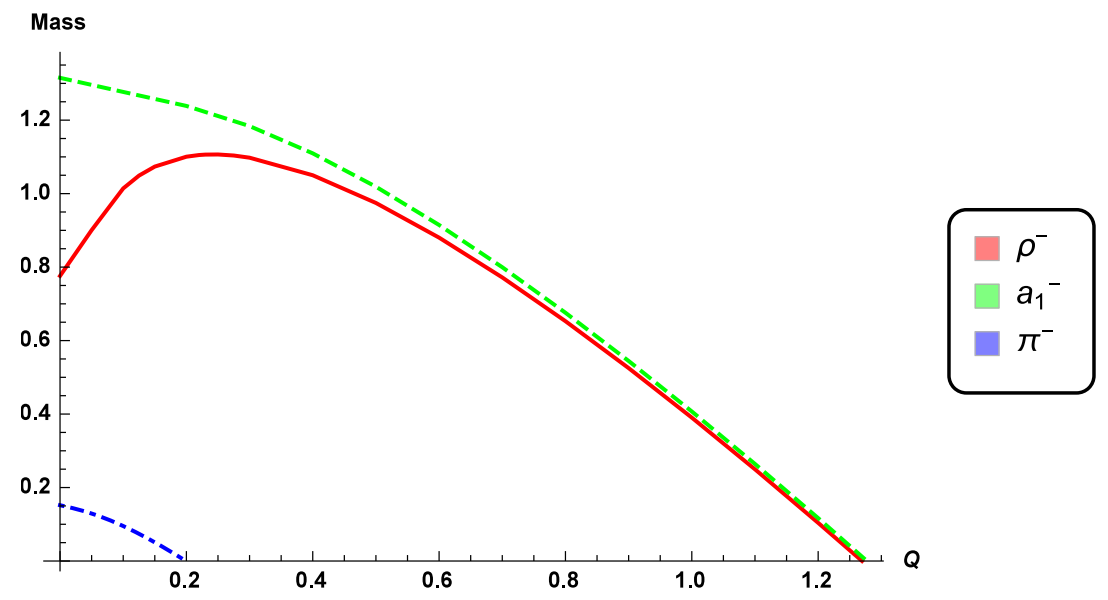

Figure 1. Density dependence of mass spectra of negative Mesons: the mass is defined as $w$ at $p=0 . \alpha=1 / 2$ and mass in GeV units. there are critical density for vanishing meson mass. red line represents for $\rho^{-}$, green dashed line for $a^{-}$, and blue dot-dashed line for $\pi^{-}$.

when setting $p=0$ as in [17]. We will use this definition of the mass in this work. Then, the equations of motion for the $\rho$ vector mesons are given by

$$
\begin{aligned}
& 0=\partial_{z}\left(\frac{f}{z} \partial_{z} \tilde{\rho}_{n}^{0}\right)+\frac{1}{z f}\left(w_{0}^{2}-f p^{2}\right) \tilde{\rho}_{n}^{0}, \\
& 0=\partial_{z}\left(\frac{f}{z} \partial_{z} \tilde{\rho}_{n}^{ \pm}\right)+\frac{1}{z f}\left[\left(w_{ \pm} \mp \bar{V}_{t}^{3}\right)^{2}-f p^{2}\right] \tilde{\rho}_{n}^{ \pm},
\end{aligned}
$$

where all fields $\tilde{\rho}^{\alpha}$ with $\alpha=0$ or \pm are subject to boundary conditions so that Dirichlet boundary conditions $\tilde{\rho}^{\alpha}(z=0)=0$ are imposed at UV to apply the gauge/gravity duality and the Neumann boundary conditions $F_{\mu z}\left(z=z_{\mathrm{IR}}\right)=0$ at the wall. In fact, the IR boundary condition at $z=z_{\mathrm{IR}}$ is chosen in a gauge-invariant way, but not unique and is made for simplicity as in [4].

Before numerically solving these boundary value problems on the constant density, i.e. the fixed $Q$ and $\alpha$, we need to recall the density dependence of the Hawking-Page (or deconfinement phase) transition in dense medium [17].

The critical density at which point the Hawking-Page transition occurs is affected by the background density, which is controlled by the parameters $Q$ and $\alpha$. For example, the calculated value of the critical density for the Hawking-Page transition reads $Q_{c r i t}=0.167$ at $\alpha=0.5$ [17]. To study mesons in the confining phase, it is necessary to keep the density parameter $Q$ below $Q_{c r i t}$ while $\alpha$ fixed. At this range, there is no pion condensate [17] because the critical density for the condensation of the negatively-charged pion is $Q_{\pi}=$ 0.199 in the figure 1 , which is bigger than $Q_{c r i t}$. In this paper, we fix $\alpha=0.5$ and either $Q=0.1$ or $Q=0.01$.

We begin to investigate dispersion relations of rho mesons. By increasing the spatial momenta, we can numerically solve the equations of motion with the prescribed boundary conditions to get energy value of the rho meson. Then, the energy values at corresponding spatial momenta will be obtained as a result, which is shown in the figure 2 . The energies 


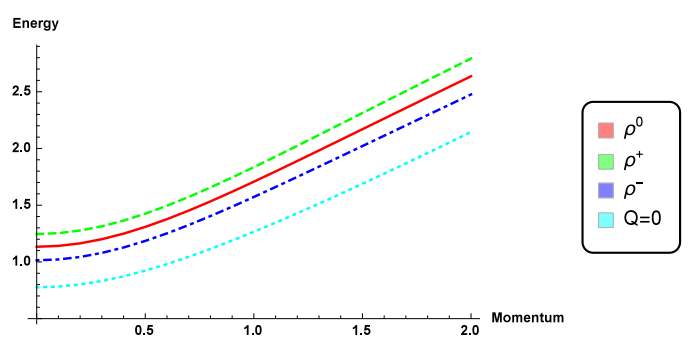

(a) Dispersion relation in the dense medium: red real line represents for $\rho^{0}$, green dashed line for $\rho^{+}$, blue dot-dashed line for $\rho^{-}$, and cyon dotted line for mesons at $Q=0$.

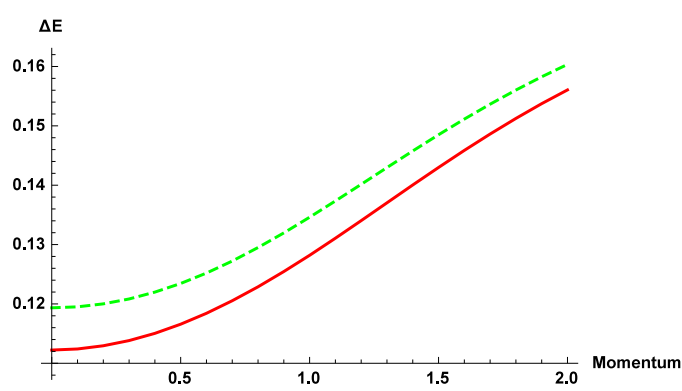

(b) Energy Differences in the dense medium: red line is energy difference of $\rho^{+}$and $\rho^{0}$, and green dashed line one of $\rho^{0}$ and $\rho^{-}$

Figure 2. Dispersion relations of the $\rho$ mesons at $Q=0.1$ and $\alpha=0.5$.

of $\rho$ mesons on the dense medium grow up as their spatial momenta $p$ increase. At a given momentum, the energies of neutral and charged rho mesons split and this results from the existence of the non-zero nuclear density. In general, the energy curve of the $\rho^{+}$is placed over the curve of the $\rho^{0}$ and the curve of the $\rho^{-}$is placed at the bottom. Moreover, the splitting among the curves become large in the heavier dense medium. Without the background densities, energies of all neutral and charged mesons grow up again as the momenta increase, but there is no splitting between the rho mesons. This is the expected result because we know that mesons have different masses according to their charges, at a given non-zero density. Note that masses of the neutral and the positively-charged rho meson on the dense medium are always bigger than those on the vacuum, but the case of the negatively-charged rho meson is dependent of the nuclear densities. As in the figure 1, the mass of the $\rho^{-}$on the vacuum reads $0.776 \mathrm{GeV}$, which is the same mass evaluated at the $Q=0.696$. If we consider a dense medium given by $Q \geq 0.696$, then energy curves of the negatively-charged $\rho$ 's on the vacuum and the dense medium intersect each other, because the gradients of the curves for the vacuum and the dense medium differ in the region of the higher momentum. In the regime of high spatial momenta, the energy curve of the $\rho^{-}$on a dense medium has greater gradient than that on the vacuum. But this does not happen at the confining phase, because the isospin density for the confining phase is always lower than the critical density. It is also noted that energy differences between rho mesons at a given momentum are not same but asymmetric as shown in figure 2(b).

The decay constant of the vector meson can be defined by calculating the currentcurrent correlator in the gague/gravity duality [4, 11]. By using the fact that Green's function of bulk gauge field can be expanded in resonances, the vector current-current correlator is derived as a sum over rho mesons [4, 34], which can be explicitly shown in [11] by incorporating the Kneser-Sommerfeld expansion with the known exact solutions. The suggested form of the decay constant is given by

$$
F_{\rho}^{2}=\left.\frac{1}{g} \frac{\partial_{z} \rho}{z}\right|_{z=\epsilon \rightarrow 0},
$$




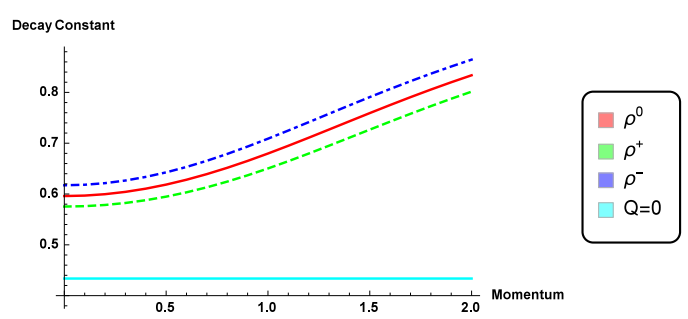

(a) Decay constants in the dense medium: while the red real line represents for $\rho^{0}$, the green dashed line for $\rho^{+}$, the blue dot-dashed line for $\rho^{-}$at $Q=0.1$, but the cyon dotted line for rho mesons at $Q=0$.

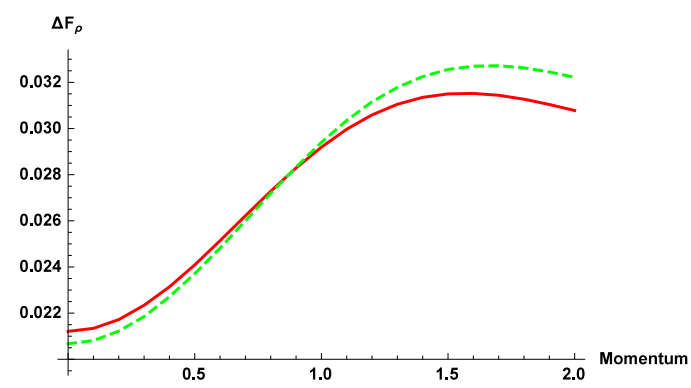

(b) Difference of Decay constants in the dense medium: the red line is decay-constant difference of $\rho^{+}$and $\rho^{0}$, and the green dashed line one of $\rho^{0}$ and $\rho^{-}$.

Figure 3. Decay constants of the $\rho$ mesons at $Q=0.1$ and $\alpha=0.5$.

where the normalized rho vector field is used. We evaluate the formula numerically and the results are presented in the figure 3. In the case of the vacuum, decay constants of all the rho mesons have the same value and are independent of their spatial momenta. But, on the dense medium, the non-trivial momentum-dependence of the decay constant is developed. Like in the case of the energy growth, the value of the decay constant becomes larger as the spatial momenta grow up. Decay constants of the neutral and the charged rho mesons have different values and they split more widely as $Q$ increases. Among the curves of the decay constants, the curve for the $\rho^{-}$is located at the top and the curves for the $\rho^{0}$ and the $\rho^{+}$are followed successively. At a glance, it seems to be peculiar that the decay constant of the more energetic $\rho^{+}$meson is smaller than that of the less energetic $\rho^{-}$meson. The naive explanation for this may be given by the dependence of the transition rate on the chemical potentials, correspondingly on the background of excessive isospin chemical potential. Note that the parameter $\alpha=0.5$ represents the isospin matter consisting of $75 \%$ proton and 25\% neutron. This excessive background charge makes the decay of a meson of the same charge slow and the decay constant of the negatively-charged rho-meson is larger than that of the positively-charged rho-meson.

\section{Dispersion relation and decay constant of the $a_{1}$ meson}

As like as the case of the vector gauge field, we consider the lowest mode of the axialvector gauge field and identify the transverse parts of the decomposition (2.19) as spin-1 $a_{1}$ mesons by field-redefinitions,

$$
a_{1 m}^{0}=\bar{a}_{m}^{3} \quad \text { and } \quad a_{1 m}^{ \pm}=\frac{1}{\sqrt{2}}\left(\bar{a}_{m}^{1} \pm i \bar{a}_{m}^{2}\right) .
$$

In terms of these, the action governing the $a_{1}$ axial-vector mesons is given by

$$
\begin{aligned}
4 g^{2} \cdot \mathcal{L}_{a}= & \partial_{M} a_{1 n}^{0} \partial^{M} a_{1}^{0 n}+4 g^{2} \phi^{2} a_{1 m}^{0} a_{1}^{0 m} \\
& +2\left\{\partial_{M} a_{1 n}^{+} \partial^{M} a_{1}^{-n}+\left(\bar{V}_{t}^{3} \bar{V}^{3 t}+4 g^{2} \phi^{2}\right) a_{1 m}^{+} a_{1}^{-m}\right. \\
& \left.+i \bar{V}_{t}^{3}\left(a_{1 m}^{+} \partial^{t} a_{1}^{-m}-a_{1 m}^{-} \partial^{t} a_{1}^{+m}\right)\right\} .
\end{aligned}
$$


The equations of motion are easily written in the four-dimensional momentum space by

$$
\begin{aligned}
& 0=\partial_{z}\left(\frac{f}{z} \partial_{z} \tilde{a}_{1 n}^{0}\right)+\frac{1}{z f}\left(w_{0}^{2}-f p^{2}\right) \tilde{a}_{1 n}^{0}-\frac{4 g^{2} \phi^{2}}{z^{3}} \tilde{a}_{1 n}^{0} \\
& 0=\partial_{z}\left(\frac{f}{z} \partial_{z} \tilde{a}_{1 n}^{ \pm}\right)+\frac{1}{z f}\left[\left(w_{ \pm} \mp \bar{V}_{t}^{3}\right)^{2}-f p^{2}\right] \tilde{a}_{1 n}^{ \pm}-\frac{4 g^{2} \phi^{2}}{z^{3}} \tilde{a}_{1 n}^{ \pm}
\end{aligned}
$$

The equations of the charged $a_{1}$ mesons contain a combination of the energy $w_{ \pm}$and the density parameters. This combination causes splitting of the physical quantities in the charged $a_{1}$ mesons such as curves in the dispersion relations and the decay constants. Actually, there exist similar terms in the equations of the motion of charged mesons and this effect can be easily seen without spatial momenta. In comparison with the $\rho$-meson case, the same numerical method is applied to the system of the axial-vector mesons. Again, we impose the Dirichlet boundary condition at the U.V. and the Neumann boundary condition at the I.R. in a gauge-invariant way.

We analyze non-trivial dispersions of the $a_{1}$ mesons on the dense medium. As in the $\rho$ mesons, the similar results are obtained and presented in the figure 4 . The curves for the dispersion relation on the dense medium are located upper than the curve for the vacuum, except for the curve for $a_{1}^{-}$meson. The intersection of the dispersion curves in the negatively-charged mesons always exists. But this occurs in a density-independent way for the $a_{1}^{-}$and $\pi^{-}$mesons but in density-dependent way for the $\rho^{-}$meson. As explained in the section of the $\rho$ meson, the energy of the $a_{1}^{-}$meson on the dense medium becomes large than those on the vacuum at the high momentum region.

At this point, we compare the curves between the vector and the axial-vector mesons in the figure 5. The curves for the vector and the axial-vector mesons of the same charge approach each other in the large momenta region. This coincidence implying the same asymptotic behaviour in large momentum region was also observed in $[4,12]$, just as expected from the equations of motion.

Analyzing decay constants of the axial-vector mesons are done likewise in the case of the $\rho$ meson. Numerically, we evaluate the same formula for the decay constant of the vector meson, in this case with the vector field replaced by the normalized $a_{1}$ fields. The result is shown in the figure 6 . Without the isospin chemical potentials, the decay constants of all the $a_{1}$ mesons have the same constant value, which is bigger than the decay constants of the $\rho$ mesons. However, on the dense medium, the decay constants of the $a_{1}$ mesons begin to grow up as $p$ increases. The $a_{1}^{+}$meson has the smallest value of the decay constant compared to the others, which is same phenomena as in the case of the rho mesons.

Like in the case of dispersion relation, we compare the decay constants of the vector and the axial-vector mesons of the same charge in figure 7. As in the dispersion relation of the energy, the curves of the decay constants for the $\rho$ and the $a_{1}$ meson of the same charge approach each other in the region of the large spatial momenta. At large $p^{2}$ region, the asymptotic values of decay constants are of the similar order. Moreover, in each case, the decay constant of the rho meson is smaller than that of the $a_{1}$ meson of the same charged type, as expected. 


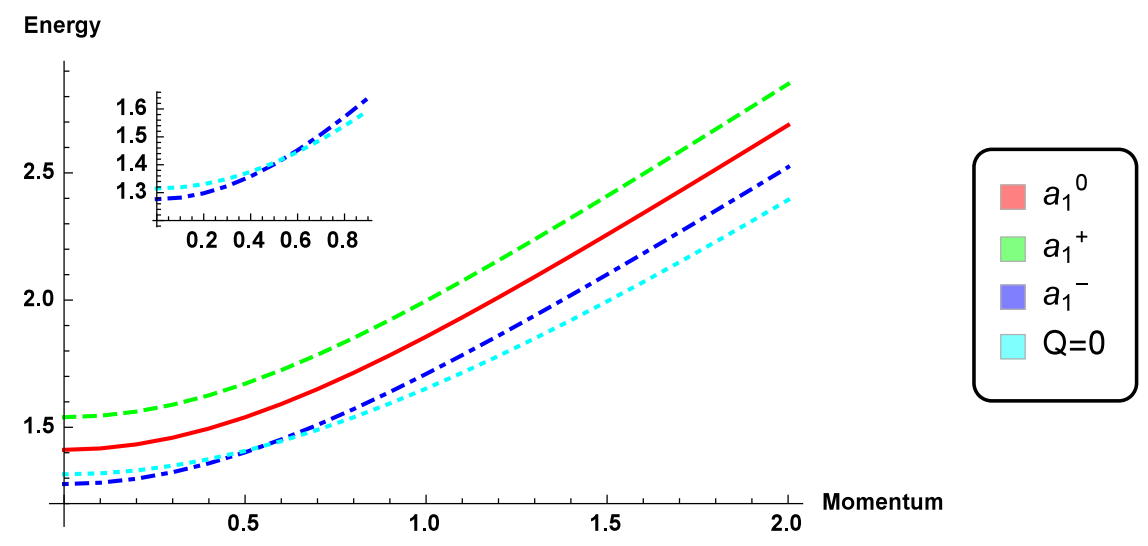

(a) Dispersion relation in the dense medium: red real line represents for $a_{1}^{0}$, green dashed line for $a_{1}^{+}$, blue dot-dashed line for $a_{1}^{-}$, and cyon dotted line for mesons at $Q=0$.

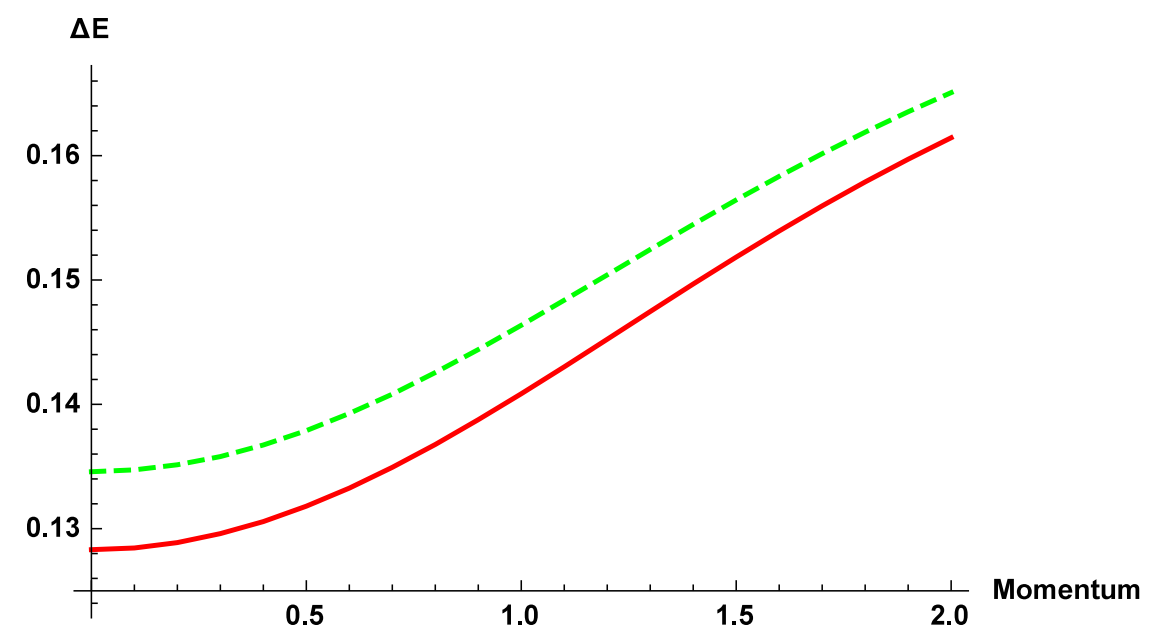

(b) Energy Differences in the dense medium: red line is energy difference of $a_{1}^{+}$ and $a_{1}^{0}$, and green dashed line one of $a_{1}^{0}$ and $a_{1}^{-}$.

Figure 4. Dispersion relation of $a_{1}$ mesons at $Q=0.1$ and $\alpha=0.5$.

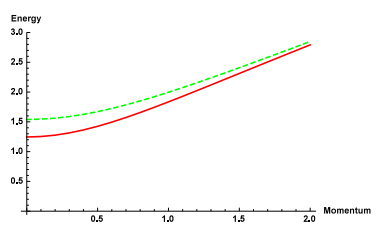

(a) Dispersion of the positive mesons.

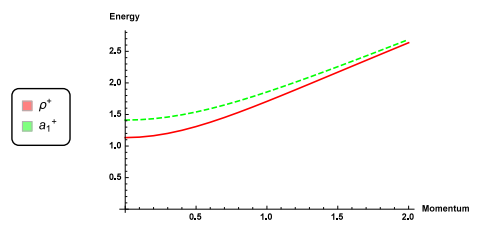

(b) Dispersion of the neutral mesons.

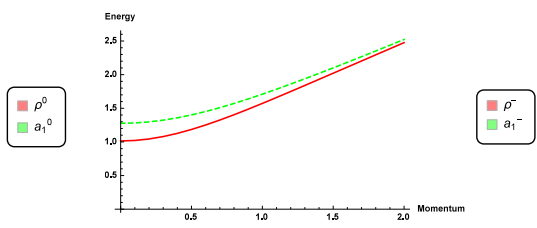

(c) Dispersion of the negative mesons.

Figure 5. Dispersion relation of $\rho$ and $a_{1}$ mesons of the same charge at $Q=0.1$ and $\alpha=0.5$.

\section{Dispersion relation and decay constant of the $\pi$ meson}




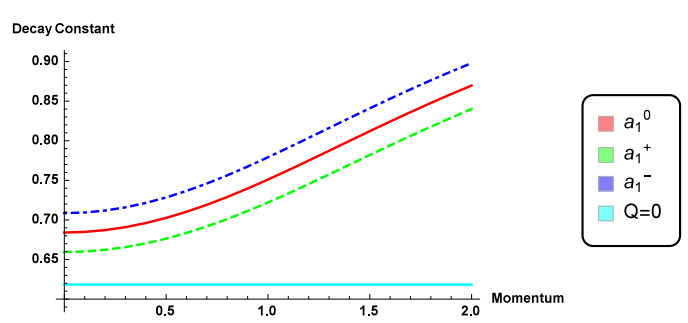

(a) Decay constants in the dense medium: the red real line represents for $a_{1}^{0}$, the green dashed line for $a_{1}^{+}$, the blue dot-dashed line for $a_{1}^{-}$on the dense medium, but the cyon dotted line for mesons at $Q=0$.

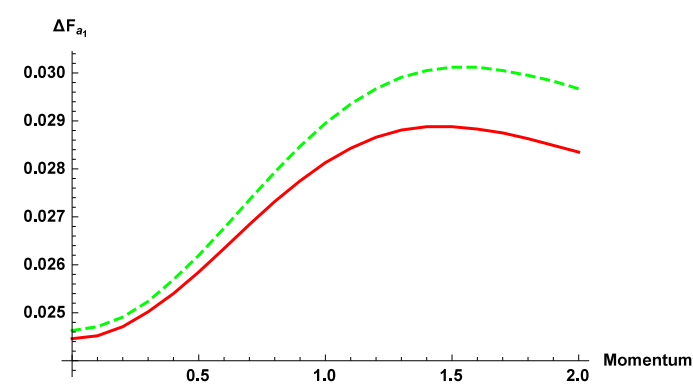

(b) Difference of Decay constants in the dense medium: the red line is decay-constant difference of $a_{1}^{+}$and $a_{1}^{0}$, and the green dashed line one of $a_{1}^{0}$ and $a_{1}^{-}$

Figure 6. Decay constants of the $a_{1}$ mesons at $Q=0.1$ and $\alpha=0.5$.

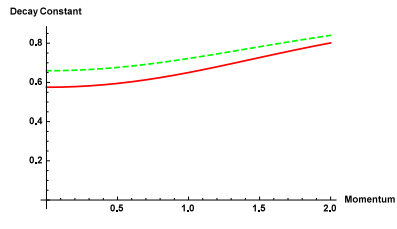

(a) Decay constants of the positive mesons.

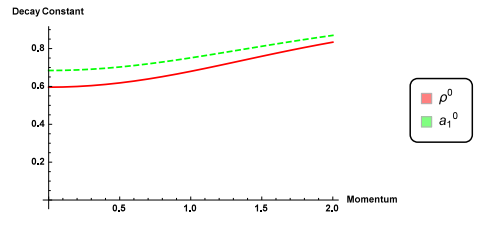

(b) Decay constants of the neutral mesons.

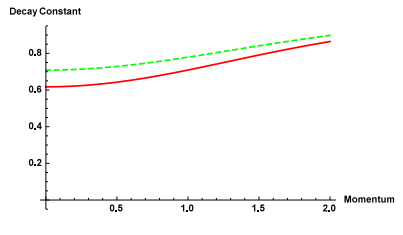

(c) Decay constants of the negative mesons.

Figure 7. Decay constants for $\rho$ and $a_{1}$ mesons of the same charge.

To interpret the lowest modes of the scalar fields as the $\pi$ meson, we make field-redefinitions given by

$$
\begin{array}{ll}
\pi^{0}=\pi^{3}, & \pi^{ \pm}=\frac{1}{\sqrt{2}}\left(\pi^{1} \pm i \pi^{2}\right), \\
\chi^{0}=\chi^{3}, & \chi^{ \pm}=\frac{1}{\sqrt{2}}\left(\chi^{1} \pm i \chi^{2}\right) .
\end{array}
$$

Then, the Lagrangian for the pseudo-scalar fields is recasted by

$$
\begin{aligned}
4 g^{2} \cdot \mathcal{L}_{\chi \pi}= & \partial_{z} \partial_{\mu} \chi^{0} \partial^{z} \partial^{\mu} \chi^{0}+4 g^{2} \phi^{2} \partial_{\mu} \chi^{0} \partial^{\mu} \chi^{0}+2 \partial_{z} \partial_{\mu} \chi^{+} \partial^{z} \partial^{\mu} \chi^{-} \\
& +8 g^{2} \phi^{2} \partial_{\mu} \chi^{+} \partial^{\mu} \chi^{-}+2 \bar{V}_{t}^{3} \bar{V}^{3 t} \partial_{m} \chi^{+} \partial^{m} \chi^{-}+4 g^{2} \phi^{2} \partial_{M} \pi^{0} \partial^{M} \pi^{0} \\
& +8 g^{2} \phi^{2}\left\{\partial_{M} \pi^{+} \partial^{M} \pi^{-}+\bar{V}_{t}^{3} \bar{V}^{3 t} \pi^{+} \pi^{-}\right\} \\
& +8 g^{2} \phi^{2}\left\{i \bar{V}_{t}^{3}\left[\pi^{+} \partial^{t}\left(\pi^{-}-\chi^{-}\right)-\pi^{-} \partial^{t}\left(\pi^{+}-\chi^{+}\right)\right]-\partial_{\mu} \chi^{0} \partial^{\mu} \pi^{0}\right. \\
& \left.-\left(\partial_{\mu} \chi^{+} \partial^{\mu} \pi^{-}+\partial_{\mu} \chi^{-} \partial^{\mu} \pi^{+}\right)\right\}
\end{aligned}
$$

The equations of motion with respect to the scalar sector consist of the systems of coupled second-order ordinary differential equations,

$$
\begin{aligned}
& 0=\partial_{z}\left(\frac{g^{2} \phi^{2} f}{z^{3}} \partial_{z} \tilde{\pi}^{0}\right)+\frac{g^{2} \phi^{2}}{z^{3} f}\left(w_{0}^{2}-f p^{2}\right)\left(\tilde{\pi}^{0}-\tilde{\chi}^{0}\right) \\
& 0=\partial_{z}\left(\frac{g^{2} \phi^{2} f}{z^{3}} \partial_{z} \tilde{\pi}^{ \pm}\right)+\frac{g^{2} \phi^{2}}{z^{3} f}\left[\left(w_{ \pm} \mp \bar{V}_{t}^{3}\right)^{2}-f p^{2}\right] \tilde{\pi}^{ \pm}-\frac{g^{2} \phi^{2}}{z^{3} f}\left[w_{ \pm}^{2} \mp w_{ \pm} \bar{V}_{t}^{3}-f p^{2}\right] \tilde{\chi}^{ \pm}
\end{aligned}
$$




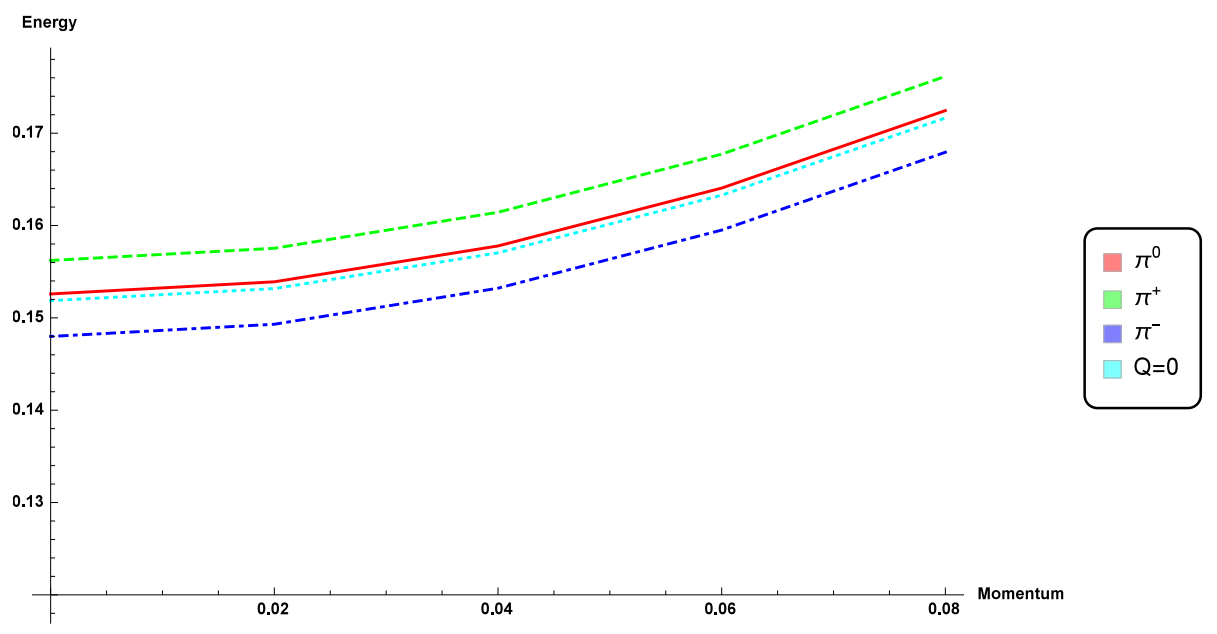

(a) Dispersion relation in the dense medium: red real line represents for $\pi^{0}$, green dashed line for $\pi^{+}$, blue dot-dashed line for $\pi^{-}$, and cyon dotted line for mesons at $Q=0$.

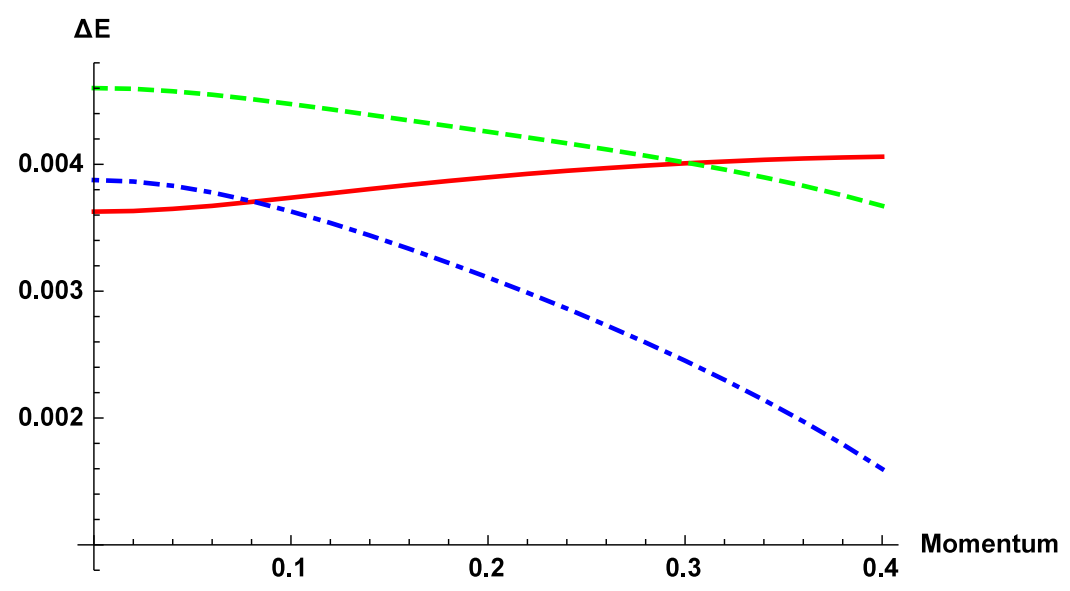

(b) Energy Differences in the dense medium: red line is energy difference of $\pi^{+}$and $\pi^{0}$, green dashed line one of $\pi^{0}$ and $\pi^{-}$, and blue dot-dashed line one of $\pi$ at $Q=0$ and $\pi^{-}$.

Figure 8. Dispersion relation of the pions at $Q=0.01$ and $\alpha=0.5$.

$$
\begin{aligned}
& \begin{aligned}
0 & \partial_{z}\left[\frac{w_{0}^{2}-f p^{2}}{z} \partial_{z} \tilde{\chi}^{0}\right]+\frac{4 g^{2} \phi^{2}}{z^{3} f}\left(w_{0}^{2}-f p^{2}\right)\left(\tilde{\pi}^{0}-\tilde{\chi}^{0}\right) \\
0=\partial_{z}\left[\frac{w_{ \pm}^{2}-f p^{2}}{z} \partial_{z} \tilde{\chi}^{ \pm}\right]+\frac{4 g^{2} \phi^{2}}{z^{3} f}\left(w_{ \pm}^{2} \mp\right. & \left.\bar{V}_{t}^{3} w_{ \pm}-f p^{2}\right) \tilde{\pi}^{ \pm} \\
& -\frac{4 g^{2} \phi^{2}}{z^{3} f}\left[w_{ \pm}^{2}-f p^{2}+\frac{\bar{V}_{t}^{3} \bar{V}_{t}^{3} z^{2}}{4 g^{2} \phi^{2}} p^{2}\right] \tilde{\chi}^{ \pm} .
\end{aligned}
\end{aligned}
$$

To solve the equations of the scalar mesons, we must specify boundary conditions, which are basically given by the same types used in the case of the vector and axial-vector mesons. We need four boundary conditions because equations of motion are given by two coupled second-order differential equations [17]. We impose two Dirichlet boundary conditions at the UV, $\tilde{\chi}^{\alpha}(0)=0=\tilde{\pi}^{\alpha}(0)$ where $\alpha$ denotes the charges 0 or \pm . Then, the 
asymptotic forms of solutions have two extra integration constants, which can be fixed by imposing two additional Neumann boundary conditions at the IR cutoff, $\partial_{z} \tilde{\chi}^{\alpha}\left(z_{\mathrm{IR}}\right)=0=$ $\partial_{z} \tilde{\pi}^{\alpha}\left(z_{\mathrm{IR}}\right)$. The numerical result with $Q=0.01$ and $\alpha=0.5$ is presented in the figure 8 . The dispersion curves of the $\pi^{+}$and the $\pi^{0}$ mesons on the dense medium sit over the curve of the $\pi^{-}$and the curve of pions at $Q=0$. As expected, the dispersion curves of the $\pi^{-}$ and the pions at the vacuum intersect each other and the energy of the negatively-charged pion on dense medium will be larger than the energy of the pions on the vacuum, seen by the blue dotted-line in the figure $8(\mathrm{c})$.

We close this section by mentioning difficulty in defining decay constants of pions. In [4], pions are considered as mixture of longitudinal part of the axial vector field $a_{\mu}^{i}$ and scalar field $\phi$. Under the chiral limit, the coupled scalar equations are simplified and it is clear what is called pion wave function. Thus, the pion decay constant can be defined by $<0\left|J_{A}^{\mu}\right| \pi(p)>=i f_{\pi} p^{\mu}$ where $J_{a}^{\mu}$ is the current for the axial vector field. But, due to the non-trivial metric factors of the dense background, it is hard to find out such a goodlooking limit at least to get a numerically calculable form. Moreover, the ground state of dual theory in dense medium is actually not well-defined.

\section{Discussion}

The hard-wall model of the holographic QCD on the dense background was studied with the help of numerical methods. By turning on the spatial momenta $p^{2}$ in the boundary space, the non-trivial behaviors of the energy dispersions and decay constants of the holographic mesons are developed. As the momenta increase, they increase and, at the same time, split due to the isospin charges. At large $p^{2}$, the asymptotic values of the energy and decay constants of the $\rho$ and $a_{1}$ mesons approach each other.

At a given $p^{2}$, the energy of positively-charged meson is always larger than the energy of the neutral and negatively-charged meson of the same type. While the energy of the positively-charged and neural mesons on the dense nuclear medium is always more large than that of the mesons on the vacuum, but the cases for the negatively-charged mesons are dependent upon the isospin chemical potential. However, the energy of the meson on the dense nuclear medium is getting bigger than that of the meson on the vacuum in the large $p^{2}$ regime.

As for the decay constants, the similar patterns were observed: the larger the spatial momenta are, the higher the decay constant of the meson is and the splitting is also existed on the dense nuclear medium. On the other hand, the value of the decay constant of the mesons on the vacuum remains literally 'constant,' irrespectively of the magnitude of the spatial momenta. We also found that, on the dense nuclear matter, the decay constant of the negatively-charged meson are always more larger than that of the positively-charged meson of the same type. This was explained by considering the decaying tendency of the charged mesons immersed in the background having finite isospin chemical potentials.

Since the dense background used for the various fluctuations was not simple, we could not have enough analytic controls and use popular approximations like the chiral limit. While the usual definitions of the QFT in the vacuum are well-known, but, in the dense 
medium, they are not well-known. So it is hard to study either the decay constant of pion or the meson form factor to investigate the interactions on the dense nuclear medium, which we leave as future works.

\section{Acknowledgments}

Bum-Hoon Lee was supported by the National Research Foundation of Korea (NRF) grant funded by the Korea government (MSIP) (2014R1A2A1A01002306). Chanyong Park was supported by Basic Science Research Program through the National Research Foundation of Korea (NRF) funded by the Ministry of Education (NRF-2013R1A1A2A10057490).

Open Access. This article is distributed under the terms of the Creative Commons Attribution License (CC-BY 4.0), which permits any use, distribution and reproduction in any medium, provided the original author(s) and source are credited.

\section{References}

[1] J.M. Maldacena, The large- $N$ limit of superconformal field theories and supergravity, Int. J. Theor. Phys. 38 (1999) 1113 [Adv. Theor. Math. Phys. 2 (1998) 231] [hep-th/9711200] [INSPIRE].

[2] E. Witten, Anti-de Sitter space and holography, Adv. Theor. Math. Phys. 2 (1998) 253 [hep-th/9802150] [INSPIRE].

[3] S.S. Gubser, I.R. Klebanov and A.M. Polyakov, Gauge theory correlators from noncritical string theory, Phys. Lett. B 428 (1998) 105 [hep-th/9802109] [INSPIRE].

[4] J. Erlich, E. Katz, D.T. Son and M.A. Stephanov, QCD and a holographic model of hadrons, Phys. Rev. Lett. 95 (2005) 261602 [hep-ph/0501128] [INSPIRE].

[5] L. Da Rold and A. Pomarol, The scalar and pseudoscalar sector in a five-dimensional approach to chiral symmetry breaking, JHEP 01 (2006) 157 [hep-ph/0510268] [INSPIRE].

[6] J. Polchinski and M.J. Strassler, Hard scattering and gauge/string duality, Phys. Rev. Lett. 88 (2002) 031601 [hep-th/0109174] [INSPIRE].

[7] J. Polchinski and M.J. Strassler, Deep inelastic scattering and gauge/string duality, JHEP 05 (2003) 012 [hep-th/0209211] [INSPIRE].

[8] I.R. Klebanov and E. Witten, AdS/CFT correspondence and symmetry breaking, Nucl. Phys. B 556 (1999) 89 [hep-th/9905104] [INSPIRE].

[9] J. Erlich, G.D. Kribs and I. Low, Emerging holography, Phys. Rev. D 73 (2006) 096001 [hep-th/0602110] [INSPIRE].

[10] S.J. Brodsky and G.F. de Teramond, Hadronic spectra and light-front wavefunctions in holographic QCD, Phys. Rev. Lett. 96 (2006) 201601 [hep-ph/0602252] [INSPIRE].

[11] H.R. Grigoryan and A.V. Radyushkin, Form factors and wave functions of vector mesons in holographic QCD, Phys. Lett. B 650 (2007) 421 [hep-ph/0703069] [INSPIRE].

[12] H.R. Grigoryan and A.V. Radyushkin, Pion form-factor in chiral limit of hard-wall AdS/QCD model, Phys. Rev. D 76 (2007) 115007 [arXiv:0709.0500] [INSPIRE]. 
[13] S. Hong, S. Yoon and M.J. Strassler, On the couplings of vector mesons in AdS/QCD, JHEP 04 (2006) 003 [hep-th/0409118] [INSPIRE].

[14] H.J. Kwee and R.F. Lebed, Pion form-factors in holographic QCD, JHEP 01 (2008) 027 [arXiv:0708.4054] [INSPIRE].

[15] Z. Abidin and C.E. Carlson, Gravitational form factors of vector mesons in an AdS/QCD model, Phys. Rev. D 77 (2008) 095007 [arXiv:0801.3839] [INSPIRE].

[16] Z. Abidin and C.E. Carlson, Gravitational form factors in the axial sector from an AdS/QCD model, Phys. Rev. D 77 (2008) 115021 [arXiv:0804.0214] [INSPIRE].

[17] B.-H. Lee, S. Mamedov, S. Nam and C. Park, Holographic meson mass splitting in the nuclear matter, JHEP 08 (2013) 045 [arXiv: 1305.7281] [INSPIRE].

[18] Y. Kim, B.-H. Lee, S. Nam, C. Park and S.-J. Sin, Deconfinement phase transition in holographic QCD with matter, Phys. Rev. D 76 (2007) 086003 [arXiv:0706.2525] [INSPIRE].

[19] B.-H. Lee, C. Park and S.-J. Sin, A dual geometry of the hadron in dense matter, JHEP 07 (2009) 087 [arXiv:0905.2800] [INSPIRE].

[20] C. Park, The dissociation of a heavy meson in the quark medium, Phys. Rev. D 81 (2010) 045009 [arXiv:0907.0064] [INSPIRE].

[21] K.B. Fadafan and E. Azimfard, On meson melting in the quark medium, Nucl. Phys. B 863 (2012) 347 [arXiv: 1203.3942] [InSPIRE].

[22] B.-H. Lee, S. Mamedov and C. Park, Nucleon mass splitting in the isospin medium, Int. J. Mod. Phys. A 29 (2014) 1450170 [arXiv:1402.6061] [INSPIRE].

[23] K. Jo, B.-H. Lee, C. Park and S.-J. Sin, Holographic QCD in medium: a bottom up approach, JHEP 06 (2010) 022 [arXiv:0909.3914] [INSPIRE].

[24] Y. Kim, Y. Seo, I.J. Shin and S.-J. Sin, Holographic meson mass in asymmetric dense matter, arXiv:1108.2751 [INSPIRE].

[25] C. Park, Holographic symmetry energy of the nuclear matter, Phys. Lett. B 708 (2012) 324 [arXiv: 1112.0386] [INSPIRE].

[26] Y. Kim, C.-H. Lee and H.-U. Yee, Holographic nuclear matter in AdS/QCD, Phys. Rev. D 77 (2008) 085030 [arXiv:0707.2637] [INSPIRE].

[27] H. Nishihara and M. Harada, Enhancement of chiral symmetry breaking from the pion condensation at finite isospin chemical potential in a holographic QCD model, Phys. Rev. D 89 (2014) 076001 [arXiv:1401.2928] [InSPIRE].

[28] H. Nishihara and M. Harada, Equation of state in the pion condensation phase in asymmetric nuclear matter using a holographic QCD model, Phys. Rev. D 90 (2014) 115027 [arXiv: 1407.7344] [INSPIRE].

[29] D.T. Son and M.A. Stephanov, QCD at finite isospin density, Phys. Rev. Lett. 86 (2001) 592 [hep-ph/0005225] [INSPIRE].

[30] A. Parnachev, Holographic QCD with isospin chemical potential, JHEP 02 (2008) 062 [arXiv:0708.3170] [INSPIRE].

[31] K.-I. Kim, Y. Kim and S.H. Lee, Isospin matter in AdS/QCD, arXiv:0709.1772 [INSPIRE].

[32] O. Aharony, K. Peeters, J. Sonnenschein and M. Zamaklar, Rho meson condensation at finite isospin chemical potential in a holographic model for QCD, JHEP 02 (2008) 071 [arXiv: 0709.3948] [INSPIRE]. 
[33] D. Albrecht and J. Erlich, Pion condensation in holographic QCD, Phys. Rev. D 82 (2010) 095002 [arXiv: 1007.3431] [InSPIRE].

[34] J. Erlich, Dualities and QCD, lectures given at $27^{\text {th }}$ Annual Hampton University Graduate Studies Program (HUGS 2012), June 4-22, Jefferson Lab, U.S.A. (2012).

[35] B.-H. Lee, C. Park and S. Shin, Holographic $1 / N_{c}$ correction from the chiral condensate, JHEP 12 (2010) 071 [arXiv: 1010.1109] [InSPIRE]. 